\title{
Fenómenos publicitarios y sociales de cambio en la dieta tradicional de México: comparativa con España y Estados Unidos
}

\section{Advertising and social phenomena of change in the traditional diet of Mexico: comparison with Spain and the United States}

Ricardo Ignacio Prado Hurtado*

Universidad Anáhuac México

Av. Universidad Anáhuac núm. 46, Col. Lomas Anáhuac, C.P. 52786, Huixquilucan, Estado de México

Editor: Rogelio del Prado Flores rprado@mostrotown.com.mx

https://orcid.org/0000-0002-4502-428X

https://doi.org/10.36105/stx.2019n3.06

\section{RESUMEN}

E n la actualidad existe un problema de salud pública en México declarado como epidemia por la Organización Mundial de la Salud (OMS): el sobrepeso, la obesidad y la diabetes. No obstante, a la luz de numerosas investigaciones se establece que parte de la resolución del problema a largo plazo será la comunicación desde la sociedad civil organizada. En este trabajo se presenta parte de una investigación en este sentido, así como avances del marco teórico y estado del arte de la misma. La metodología de este trabajo consiste en una revisión documental de la fenomenología en torno de la comunicación de dos países en comparación con México influida por los cambios de dieta producidos por fenómenos sociales y políticos. El objetivo de este trabajo de investigación es establecer coincidencias entre los puntos de vista que dan base a las campañas localmente y plantear la posibilidad de señalar un camino viable para detener la epidemia.

\footnotetext{
* Director General de Servicios Creativos en Mostrotown Publicidad, agencia especializada en contramarketing, creatividad y mercadotecnia sociales. Con más de 20 años trabajando en el medio publicitario, ha formado parte de agencias de publicidad nacionales e internacionales trabajando para cuentas en una extensa variedad de categorías de productos y servicios; ha colaborado como docente y conferencista en universidades nacionales. Desde enero de 2018, es alumno del Doctorado en Investigación de la Comunicación, Facultad de Comunicación de la Universidad Anáhuac México y becario del CONACYT.
} 
Palabras clave: publicidad con causa, alimentos ultraprocesados, campañas desde la sociedad civil, organizaciones de protección de consumidor, obesidad y diabetes.

\section{ABSTRACT}

Currently there is a public health problem in Mexico declared as an epidemic by the World Health Organization (WHO): overweight, obesity and diabetes. However, in the light of frequent research it is established that part of the long-term problem resolution will be food literacy. This paper presents part of an investigation in this regard, as well as advances in the theoretical framework and state of the art of it. The methodology of this work consists of a documentary review of the food literacy strategies of the countries compared to Mexico influenced by dietary changes produced by social and political phenomena. The objective of this research work is to establish coincidences between the views that give rise to the campaigns locally and to propose the possibility of pointing out a viable way to stop the epidemic.

Keywords: advertising with cause, ultra-processed foods, campaigns from civil society, consumer protection organizations, obesity and diabetes.

\section{INTRODUCCIÓN}

El presente trabajo contrasta a partir de una aproximación documental la evolución en cuestión de publicidad de alimentos ultraprocesados, especialmente cereales, y los cambios de dieta de tres países: España, Estados Unidos de Norteamérica y México, con una especial atención en el último, con la finalidad, desde la revisión de la literatura que explica los cambios de estilos de vida, los cambios políticos y en el caso de México de colonización, de evaluar si las campañas y estrategias de la comunicación desde las Organizaciones Protectoras del Consumidor (OPC) pueden ser un camino viable para prevenir y detener la actual epidemia de sobrepeso, obesidad y diabetes en México en el largo plazo. En el presente trabajo se expondrán puntos de vista teóricos que permitirán contrastar aspectos relacionados con la ética de la práctica publicitaria de comunicación y sus objetivos, así como la propuesta de inclusión de una nueva variedad de stakebolders que no se tomaban en cuenta en las estrategias tradicionales de la publicidad. Se expondrán también los puntos de vista históricos y contemporáneos sobre la comunicación alrededor de las 
dietas, española, estadounidense y mexicana que podrían jugar un papel fundamental en la implementación, desde la comunicación, acerca de las ventajas o desventajas de seguir un determinado tipo de dieta a través de diferentes estrategias de comunicación a lo largo del tiempo.

\section{ESTADO DEL ARTE}

Las estrategias de mercadotecnia y publicidad de las empresas productoras de Alimentos Ultraprocesados (AUP) en medios son cada vez más sofisticadas y tienen como uno de sus objetivos alcanzar consumidores en momentos cada vez más vulnerables. La influencia que ejercen sobre el estado, la academia y la ciencia, es enorme, y los consumidores se encuentran vulnerables, desprotegidos y presuntamente mal informados.

El conjunto de esfuerzos de mercadotecnia y comunicación de las multinacionales productoras de AUP tiene como principal finalidad presentar al consumidor las marcas de sus productos, los supuestos beneficios de los mismos y los estilos de vida relacionados con su consumo creando un ambiente obesogénico. El consumo regular de estos alimentos puede generar graves problemas a la salud a mediano y largo plazos, y en ocasiones pueden llegar a acortar prematuramente la vida de las personas. Es por esto que la sociedad civil ha tenido que suplir esta función, propia del Estado, a través de Organizaciones No Gubernamentales y Organizaciones de la Sociedad Civil (ONG y OSC) Protectoras de los Derechos de los Consumidores (OPC). Estas también han desarrollado estrategias de contramercadotecnia para sensibilizar, señalar y contraargumentar frente al consumidor el discurso de venta de enormes dimensiones de estas corporaciones comerciales de presencia y actividad mundial.

El presente estado del arte se encuentra dividido en dos secciones principales y tiene como objetivos revisar: en primer lugar el panorama de la publicidad de AUP en un contexto internacional. En una segunda sección se revisarán los mensajes intervinientes y estrategias de las productoras de AUP para impactar a los consumidores y cómo es que las empresas productoras de AUP logran enganchar a los consumidores con su publicidad para persuadir con múltiples estrategias al consumo de sus productos en medios, aprovechando su penetración.

Se establecieron seis categorías de análisis sobre la literatura con la finalidad de generar el contraste para detectar diferencias y similitudes entre el marco histórico de España, Estados Unidos y México con el discurso actual de las OPC que se pueden observar en la Tabla 1 que compara lateralmente las categorías por país. 
Las categorías de análisis son:

- Estudios y estrategias de las OPC: Se refiere a las acciones en diferentes niveles que lleva acabo la sociedad civil organizada para contraargumentar y señalar las prácticas negativas de las empresas multinacionales productoras de AUP.

- Contexto histórico publicitario y social definidos por país: Análisis documental de los ejemplos y tendencias más destacadas de la publicidad de AUP en los tres países analizados.

- Fenómenos sociales de cada uno de los tres países: Revisión de las coyunturas a nivel social que podrían haber influido en los cambios de dieta de los países analizados.

- Variaciones en la dieta de cada país: Revisión de las tendencias citadas en la literatura que llevaron a cada uno de los tres países analizados a modificar sus dietas tradicionales.

- Acciones del Estado y la sociedad para el cambio de dieta: Breve revisión del papel del Estado en los cambios de dieta de cada país, ya sea por acción o por omisión.

- Acciones de las OPC para señalar los problemas y posibles soluciones: Análisis de casos citados por los diferentes estudiosos de las acciones implementadas por las OPC y ONG para llevar a cabo su labor en pro del despertar de la conciencia social.

En el Cuadro 1 se muestran las categorías de análisis por país (México, España y Estados Unidos).

CUADRO 1. COMPARATIVO DE CATEGORÍAS DE ANÁLISIS POR PAÍS

\begin{tabular}{|l|l|l|l|}
\hline \multicolumn{1}{|c|}{$\begin{array}{c}\text { CATEGORÍAS DE ANÁLISIS } \\
\text { POR PAÍS }\end{array}$} & \multicolumn{1}{|c|}{ ESPAÑA } & \multicolumn{1}{c|}{ ESTADOS UNIDOS } & \multicolumn{1}{c|}{ MÉxICO } \\
\hline $\begin{array}{l}\text { Estudios y estrategias de } \\
\text { las OPC }\end{array}$ & $\begin{array}{l}\text { Organizaciones como } \\
\text { FACUA realizan estudios, } \\
\text { campañas, investigación } \\
\text { yestrategias jurídicas de } \\
\text { presión a gobiernos. }\end{array}$ & $\begin{array}{l}\text { Organizaciones como } \\
\text { CSPI son líderes en } \\
\text { investigación; realizan } \\
\text { estudios, campañas, } \\
\text { activismo y estrategias } \\
\text { jurídicas. }\end{array}$ & $\begin{array}{l}\text { Organizaciones como } \\
\text { ASA realizan estudios, } \\
\text { campañas, investigación, } \\
\text { activismo y estrategias } \\
\text { jurídicas. }\end{array}$ \\
\hline
\end{tabular}




\begin{tabular}{|c|c|c|c|}
\hline $\begin{array}{c}\text { CATEGORÍAS DE ANÁLISIS } \\
\text { POR PAIIS }\end{array}$ & ESPAÑA & ESTADOS UNIDOS & MÉXICO \\
\hline $\begin{array}{l}\text { Contexto histórico, } \\
\text { publicitario y social } \\
\text { definidos por país }\end{array}$ & $\begin{array}{l}\text { La publicidad cambia } \\
\text { de acuerdo con las } \\
\text { tendencias sociales } \\
\text { y mercadológicas; la } \\
\text { sociedad sigue las } \\
\text { tendencias. }\end{array}$ & $\begin{array}{l}\text { Las multinacionales } \\
\text { invierten fuertes } \\
\text { sumas en publicidad } \\
\text { y crean las tendencias } \\
\text { mercadológicas a nivel } \\
\text { mundial. }\end{array}$ & $\begin{array}{l}\text { Fuerte influencia de } \\
\text { Estados Unidos en } \\
\text { cuestión mercadológica. } \\
\text { La tendencia a lo } \\
\text { moderno y lo práctico } \\
\text { han rezagado la dieta } \\
\text { tradicional. }\end{array}$ \\
\hline $\begin{array}{l}\text { Fenómenos sociales } \\
\text { de cada uno de los tres } \\
\text { países }\end{array}$ & $\begin{array}{l}\text { Guerra civil española, } \\
\text { posguerra mundial, } \\
\text { sociedad altamente } \\
\text { restrictiva. }\end{array}$ & $\begin{array}{l}\text { Posguerra mundial, } \\
\text { desarrollo de grandes } \\
\text { empresas productoras. }\end{array}$ & $\begin{array}{l}\text { Degradación de la dieta } \\
\text { tradicional indígena, } \\
\text { políticas públicas } \\
\text { hacia la alimentación } \\
\text { occidentalizada. }\end{array}$ \\
\hline $\begin{array}{l}\text { Variaciones en la dieta de } \\
\text { cada país }\end{array}$ & $\begin{array}{l}\text { De lo natural a lo } \\
\text { manufacturado, de } \\
\text { lo manufacturado a } \\
\text { los claims naturales } \\
\text { y adaptaciones } \\
\text { tecnológicas. }\end{array}$ & $\begin{array}{l}\text { Tendencia a la } \\
\text { hiperalimentación, } \\
\text { posguerra y posterior } \\
\text { tendencia a la } \\
\text { alimentación saludable. }\end{array}$ & $\begin{array}{l}\text { De los cereales y proteína } \\
\text { vegetal al consumo de } \\
\text { proteína animal. }\end{array}$ \\
\hline $\begin{array}{l}\text { Acciones del Estado y la } \\
\text { sociedad para el cambio } \\
\text { de dieta }\end{array}$ & $\begin{array}{l}\text { Promoción } \\
\text { gubernamental al } \\
\text { consumo de productos } \\
\text { industrializados. }\end{array}$ & $\begin{array}{l}\text { Promoción de } \\
\text { la abundancia, } \\
\text { concientización hacia } \\
\text { ingredientes como el } \\
\text { azúcar, promoción de la } \\
\text { proteína aminal. }\end{array}$ & $\begin{array}{l}\text { Políticas públicas de } \\
\text { promoción hacia la } \\
\text { alimentación basada en } \\
\text { proteínas. Abandono de } \\
\text { los cereales endémicos. }\end{array}$ \\
\hline $\begin{array}{l}\text { Acciones de las OPC para } \\
\text { señalar los problemas y } \\
\text { posibles soluciones }\end{array}$ & $\begin{array}{l}\text { Promoción de la } \\
\text { protección al consumidor, } \\
\text { orientación científica al } \\
\text { problema, apoyo social } \\
\text { a las ONG. Promoción } \\
\text { gubernamental a } \\
\text { políticas de salud. }\end{array}$ & $\begin{array}{l}\text { Desarrollo de ONG } \\
\text { de gran calado, } \\
\text { especialización } \\
\text { de la protección al } \\
\text { consumidor. Figuras } \\
\text { gubernamentales } \\
\text { como embajadores de } \\
\text { soluciones. }\end{array}$ & $\begin{array}{l}\text { Campañas desde la } \\
\text { sociedad civil, acciones } \\
\text { legales, impuestos a los } \\
\text { AUP. }\end{array}$ \\
\hline
\end{tabular}

FUENTE: ELABORACIÓN PROPIA. 


\section{PUBLICIDAD DE ALIMENTOS ULTRAPROCESADOS EN EL MUNDO}

La comunicación publicitaria ha sido instrumento de difusión de todas estas ideas en diferentes tiempos, campos y lugares, especialmente en los países más desprotegidos (Taylor y Jacobson, 2016; Alianza por la Salud Alimentaria, 2018a; Alianza por la Salud Alimentaria, 2018b; Calvillo A., 2018), los de ingreso medio y bajo donde ha sido más rentable y sencillo diseminar y validar conceptos que no necesitan ser, ni siquiera, comprobados científicamente, sino meros instrumentos de persuasión de grandes multinacionales (Aguilar, 2008; Alianza por la Salud Alimentaria, 2018; Arragán, Guerra, y Villalpando, 2017) con características de megalomanía que utilizan estratégicamente sus medios para modelar las pasiones humanas en lugar de cultivar en intelecto a las personas (Del Prado, 2016) y eso es exactamente lo que parecieran hacer las diez empresas multinacionales que controlan el mercado mundial de alimentos (Alianza por la Salud Alimentaria, 2018; Calvillo, 2018).

\section{MENSAJES INTERVINIENTES DE OPC}

En esta sección se lleva a cabo una revisión de autores que analizan los mensajes intervinientes desde las ONG en contra de la publicidad de alimentos chatarra y ambiente obesogénico (Alianza por la Salud Alimentaria, 2018; Espinosa, 2017), así como los efectos de sensibilización que se alcanzan en la sociedad. Las metodologías usadas en las fuentes son muy diversas, en el caso de la OPS (Organización Panamericana de la Salud, 2015) se usó información de la base de datos Passport sobre los mercados mundiales, publicada en Internet por Euromonitor International y el sistema NOVA de clasificación de alimentos (Organización Panamericana de la Salud, 2015). Aquí se ofrece un panorama muy amplio y divide los alimentos ultraprocesados en: bebidas gaseosas, cereales para el desayuno, snacks dulces y salados, golosinas (caramelos y chocolates), helados, galletas, jugos de frutas y verduras, bebidas deportivas y energizantes, productos para untar, salsas y comidas preparadas. Además de productos de venta en establecimientos de comida rápida (Organización Panamericana de la Salud, 2015; Popkin, 2012; Robinson, 2007). También utilizan análisis correlacionales con la finalidad de clasificar las ventas de AUP de acuerdo con tres factores sociales y económicos que son: la urbanización, el crecimiento y la desregulación de los mercados (Taylor y Jacobson, 2016; Organización Panamericana de la Salud, 2015). En este estudio se analizaron 80 países.

En el caso del informe El ambiente escolar obesogénico en México: La necesidad de mejorar la regulación de la venta de alimentos y bebidas en escuelas, que se realizó entre junio y julio de 
2017 en nueve escuelas públicas primarias en la Ciudad de México, Cuernavaca y San Luis Potosí se dirigieron a actores clave: autoridades de salud y educativas; personal directivo, docente y de venta; miembros de comités y estudiantes (Alianza por la Salud Alimentaria, 2018). Establece, entre otros datos, que uno de cada tres niños en México vive con obesidad y que uno de cada dos niños nacidos a partir del 2010 desarrollará diabetes a lo largo de su vida, convirtiéndose en una situación de gran urgencia (Alianza por la Salud Alimentaria, 2018). Sin embargo, esta información no ha bastado para que el gobierno legisle a favor del bien mayor del individuo (Turky Ernest, 2015; Sorscher, 2018; Schwalb, Sanborn, Galarza, Garcia, y Kogan, 2014). Las campañas de sensibilización tienen diferentes niveles de impacto en jóvenes a nivel mundial, de acuerdo con los avances en diferentes áreas por país. En Estados Unidos los jóvenes tienen una mayor conciencia sobre temas de obesidad y consumo de cereales, mientras que en Colombia y México por otro lado (Calvillo, 2018) los cereales en el desayuno siguen siendo una opción considerada como saludable en comparación con muchas otras opciones alimenticias (Organización Panamericana de la Salud, 2015; World Health Organization, wHO, 2014). Chile es un país donde se tiene identificado que el cereal es básicamente comida "chatarra" además de que un elemento sumamente eficiente para la venta de estos productos es el uso de personajes dirigidos a niños y adolescentes como son las mascotas, por lo que después de una batalla legal de varios años, se han prohibido en los empaques de cereal de caja (Alianza por la Salud Alimentaria, 2018; Taylor y Jacobson, 2016; Barragán, Guerra, y Villalpando, 2017; Brown, 2014). La respuesta de la industria de Cereales Ultraprocesados (CUP) ha sido una lucha feroz para proteger su mercado ya que en este país los intereses corporativos han logrado retrasar la aprobación de las leyes a este respecto por casi diez años, siendo indispensables los cabilderos en las cámaras para darse a la tarea de sabotear las audiencias (Jacobs, 2018).

Las estrategias de mercadotecnia y publicidad de las empresas productoras de Cereales Ultraprocesados (CUP) en medios electrónicos son cada vez más sofisticadas (Taylor y Jacobson, 2016; Calvillo, 2018; El Poder del Consumidor, 2018), con el objetivo de atrapar a los consumidores en momentos cada vez más vulnerables, como pueden ser la vía pública, las escuelas, los eventos deportivos y recreativos, entre otros. La influencia que ejercen sobre el Estado, la academia y la ciencia, es enorme, y los consumidores se encuentran vulnerables, desprotegidos y en ocasiones mal informados; también se mandan a hacer investigaciones a modo de las multinacionales para permear en la opinión pública información favorable para sus productos de forma falaz (Calvillo, 2018). El conjunto de esfuerzos de las empresas de CUP, cuyo objeto es rodear al consumidor de comunicación comercial en todas las plataformas posibles, se denomina ambiente obesogénico (Espinosa, 2017). Es por esto que la 
sociedad civil ha tenido que suplir esta función propia del Estado a través de Organizaciones No Gubernamentales (ONG) y Organizaciones Protectoras de los Derechos de los Consumidores (OPC) (Taylor, 2016). Éstas también han desarrollado estrategias para sensibilizar, señalar y contraargumentar, frente al consumidor, el discurso de enormes dimensiones de las corporaciones comerciales.

Los autores coinciden en prácticamente todos los estudios en el peligro relacionado con el consumo de alimentos y bebidas ultraprocesadas, ya que según señalan éstas pueden causar diferentes enfermedades entre ellas diabetes, obesidad, síndrome metabólico y algunos tipos de cáncer (Taylor y Jacobson, 2016; Aguilar, 2008; Alianza por la Salud Alimentaria, 2018; Baraybar, Baños, Barquero, Goya, y De la Morena, 2017; Canal Diabetes, 2018). También se encuentran coincidencias en el hecho de que la publicidad y la mercadotecnia generan compra condicionada con herramientas como el uso de personajes, regalos, promociones, etc. (Taylor y Jacobson, 2016; Calvillo, 2018; Macari, 2018; Nestlé, 2002). Y en cómo genera un tipo de manipulación sobre los grupos desprotegidos como los niños, mientras que en grupos jóvenes pueden generar rechazo (Calvillo, 2018). En varios de los artículos y reportes se menciona la necesidad de crear más y mejores leyes para la protección del consumidor y la urgencia de que el Estado reconozca que nos encontramos frente a una epidemia de salud muy grave especialmente en México y demás países de medio y bajo ingreso, mercados donde estas empresas obtienen sus mayores dividendos (Nestlé, 2002; Olivares, 2018; Organización Panamericana de la Salud, 2015; Taylor y Jacobson, 2016; The Public Health Advocacy Institute, 2017).

\section{MARCO TEÓRICO Y CONTEXTUAL}

\section{Cambio de dieta y de estilos de vida}

Elfenómeno ha tenidolugar históricamente en varios países para dar paso a la comunicación de una supuesta nueva dieta, al parecer más moderna, práctica y adecuada a los estilos de vida modernos. Es así como las dietas tradicionales ha sufrido cambios radicales de acuerdo con los procesos históricos que han sucedido en España, Estados Unidos de Norteamérica y México de acuerdo con diferentes fenómenos históricos, sociales, comerciales y políticos, como puede ser el cambio de tradiciones probablemente relacionadas con la guerra civil española, la cultura del racionamiento posterior a la Segunda Guerra Mundial en Estados Unidos y el caso de los prejuicios de los colonizadores españoles acerca de un supuesto rezago en los habitantes de Mesoamérica debido a su dieta. 


\section{Caso España}

En España el fenómeno se ubica desde la década de los años sesenta ya que Arribas Macho describe con sus estudios los periodos previos a la industrialización utilizando la publicidad como unidad de análisis (Arribas, 1996). Pero son los trabajos de Mabel Gracia los pioneros en analizar la publicidad alimentaria relacionada con discursos sobre alimentación en sus estudios de la misma de 1960 a 1990 (Gracia, 1996).

En la publicidad de este país se observan modelos que se relacionan con la salud, el bienestar y la comida en cada momento histórico (Días y González, 2013). Se observa también que los valores han cambiado y que al consumidor se le ha sesgado históricamente respecto al estilo de vida que cada comida demanda ya que la respuesta a la publicidad por parte del consumidor no es mecánica (Días y González, 2013). En las décadas de los años sesenta y setenta es cuando la publicidad empieza a presentar una orientación hacia la industrialización, aun cuando el 40\% de la población trabajaba en el sector agrario.

La comunicación publicitaria busca adiestrar a los consumidores en la compra de productos manufacturados. Se utilizan palabras como: sano, puro o natural para que no fueran rechazados los productos industrializados. Se les da la connotación de "extranjeros" a varios alimentos para generar la imagen de modernidad en la mente de los consumidores. Las marcas se convierten en una garantía de calidad. Ya en la década de los años ochenta la audiencia está compuesta por la primera generación “adiestrada” y es cuando en España los productos industrializados se expanden a las masas. La saturación de marcas se hace evidente y empiezan a llevar con publicidad a la diferenciación por medio de nuevas presentaciones para la diversidad de consumidores (Rodríguez y Sanz, 1992 citados en Días y González, 2013). Se aprueba la Ley General de Publicidad.

Rapidez y facilidad se convierten en dos de los atractivos más usados por la comunicación comercial. Fenómenos como el comúnmente conocido de "las vacas locas" hacen que en la década de los años noventa las marcas empiecen a responder al consumidor de forma más sensible, dar entrada a las asociaciones de consumidores. "El discurso busca recuperar la confianza del consumidor. Las grandes empresas toman la iniciativa de crear institutos de alimentación que respalden sus estrategias publicitarias y la posición de sus productos en el mercado nacional” (Días y González, 2013). En la primera década del 2000 las marcas españolas dan prioridad a la salud como tema publicitario; por ejemplo, si los expertos recomiendan comer frutas y verduras diariamente, las empresas buscan ofrecerlas en un práctico envase, o si la ciencia opina que el colesterol malo puede bajar gracias a los componentes de los peces de agua fría, la industria ofrece alimentos que concentran en una sola ración todos los beneficios o añaden omega 3 a la leche o antioxidantes a la fruta (Días y González, 2013). Hacia finales del 2010 la publicidad de alimentos se especializaba y buscaba con sus textos 
matizar las propuestas científicas en conjunto con textos sobre naturaleza y practicidad. Actualmente, las marcas de alimentos orientan su comunicación a las causas sociales ya que esta se presenta como una megatendencia en Europa, Estados Unidos y Australia a la que las personas reaccionan muy positivamente y dando preferencia a estas (Días y González, 2013).

El marketing social se ha convertido en estos países en un estandarte muy útil para la publicidad de las marcas de alimentos, sin embargo, estos temas de Responsabilidad Social Empresarial (RSE) no necesariamente están vinculados a las necesidades reales de sus consumidores. Así es como, de acuerdo con estudios globales de OPC o de ONG las marcas se cubren con un manto de responsabilidad social sin atender a los efectos que pueden causar sus fórmulas alimenticias en la salud de la población (Taylor, 2016).

La mayoría de los autores reconocen que, históricamente, el marketing social inició con las campañas de Wiebe en 1952 apoyado en su célebre cuestionamiento sobre "¿Por qué no puede venderse la solidaridad como se vende el jabón?”, solo respondido en 1971 por Kotler y Zaltman (1971, pp. 3-12) y más recientemente por Andrea-sen (1996, pp. 108-114) que afirma que el marketing social es la aplicación de la tecnología para planear, analizar, ejecutar y evaluar estrategias diseñadas para modificar el comportamiento de las personas para mejorar el bienestar social y personal (citados en Días y González, 2013).

\section{Caso Estados Unidos}

En Estados Unidos desde principios del siglo XX, la ingesta inadecuada de calorías y nutrientes de los estadounidenses llevó a la publicidad a invitar a la gente a comer más y en una mayor variedad lo que llevaría a la sociedad de Estados Unidos a la sobrenutrición y, por consiguiente, a la publicidad a dirigir sus esfuerzos a promover el mensaje de comer menos. La comunicación comercial desde el gobierno empezó a sugerir en la década de los ochenta que el exceso de azúcar era dañino, únicamente en personas con caries. Las multinacionales de alimentos y bebidas en Estados Unidos llevaron a una sociedad de consumo donde pasaron de las pequeñas granjas a los gigantes corporativos, de cocinar en casa a consumir más de la mitad de sus alimentos fuera de casa y de consumir comida "entera" producida cerca de casa a consumir una gran cantidad de productos procesados fuera del país y transportados grandes distancias (Nestle, 2002). La publicidad de alimentos ha seguido estas tendencias influenciada poderosamente por la economía del país que ha forzado a las empresas a crecer de forma gigantesca.

En Estados Unidos la gente ha sufrido una "transición nutricional" de acuerdo con su bienestar y empiezan a abandonar una dieta con base en plantas y cereales y migran a una 
donde comen más carne, grasa y alimentos procesados. Esto ha llevado a las industrias a comercializar marcas donde el presupuesto de mercadotecnia es mucho mayor al presupuesto de producción, lo que ha tenido como principal consecuencia que los estadounidenses inviertan en promedio menos de $10 \%$ de su sueldo en alimentos. La práctica de comer fuera de casa en Estados Unidos tuvo un incremento de casi el doble histórico en las décadas de los años ochenta y noventa con el desarrollo de alimentos biperconvenientes con presupuestos en publicidad extraordinarios que se mantienen hasta nuestros días. Desde las empresas pequeñas hasta las enormes multinacionales estadounidenses invierten fuertes sumas en crear la imagen y promover el consumo, por ejemplo.

McDonald's invirtió 627.2 mdd, Burger King 403.6 mdd, Taco Bell 206.5 mdd y Coca y Coca de dieta 174.4 mdd en medios directos de publicidad hablando de mutinacionales, pero también pequeños productores tuvieron presupuestos de publicidad impresionantes y para muestra un botón, 117 mdd para la goma de mascar Wrigley's, y cerca de 80 mdd en dulces M\&M’s (Nestle, 2002).

\section{Caso México}

En el caso de México, las causas son mucho más complejas que en otros países, especialmente los desarrollados. Desde la conquista española en México se crea un prejuicio desde la comunicación contra la dieta tradicional de los pueblos mesoamericanos.

Globalmente se dio una transformación de los sistemas nacionales de alimentación en la posguerra que ha llevado a cambios de dieta por todo el mundo. La distribución globalizada de tecnología para producir, transportar o comercializar alimentos, así como el flujo de capital y servicios, han provocado que los precios de las grasas vegetales, azucares y alimentos de origen animal se hayan reducido, proceso acompañado por la difusión de mensajes en medios masivos (Popkin, Adair y Ng, 2012). Aunque el impacto de los cambios ambientales en el ámbito alimentario ha sido global, se reconocen algunas particularidades de los países menos industrializados (entre los que se cuenta México) que no permiten derivar las implicaciones de dichos cambios a partir de la experiencia de los países más ricos (Espinosa, 2017).

Los gobiernos coloniales en México rechazaron sistemáticamente la dieta de los habitantes, prácticamente desde que llegaron, ya que notaron diferencias importantes entre ellos y los conquistados, que atribuyeron a la alimentación. El alimento más satanizado fue el maíz, ya que constituía la base de la dieta de los pueblos originarios. Se generó entonces el prejuicio de que la aparente falta de desarrollo físico, tecnológico y cultural era producto de una alimentación pobre basada en cereales de baja calidad como el maíz, en lugar del trigo, 
que fue la piedra angular de la dieta de las culturas europeas y que además no incluía carnes de origen bovino o caprino, huevo y leche (Espinosa, 2017; Rivera, Enciso, Estrada, y Fonseca, 2017; Schwalb et al., 2014; Soariano, 2008). En México, la presencia del trigo como un nuevo alimento posicionó al maíz como un elemento de identidad indígena frente a los españoles, según el historiador Jefrrey Pilcher (2001), si un indígena quería dejar de serlo, se iba a vivir a las ciudades, vestía como mestizo y comía pan, lo que era suficiente para dejar de ser reconocido como indígena (Pilcher, 2001 citado en Beltrán, 2019).

Durante el siglo XIX, era común atribuir superioridad a ciertas sociedades a partir del alimento básico consumido, en el caso de España y Estados Unidos: el trigo. Después de la Revolución mexicana personajes tan ilustres como Manuel Gamio, considerado el padre de la antropología en México y funcionario público de alto nivel, denominó a este fenómeno como la esclavitud del maíz ya que limitaba de forma considerable el desarrollo del país porque la población no se acostumbraba a sembrar ni consumir otros cereales (Beltrán, 2019).

El connotado médico posrevolucionario Alfredo Ramos, por ejemplo, argumentaba que la historia enseña cómo los pueblos mejor alimentados, con alimentación variada y completa, eran generalmente los conquistadores de los que se alimentaban de maíz y yerbas (citado en Pío, 2013).

El mismo Francisco Miranda (fundador del Instituto Nacional de Nutriología, INN, que llegaría a ser el actual Instituto Nacional de Ciencias Médicas y Nutrición Salvador Zubirán, INCMNSZ), expresaba de forma prejuiciosa en 1946 que un sujeto mal alimentado se volvía flojo e incapaz de realizar trabajo de calidad o intenso, que más bien vivía indiferente de su entorno limitado físicamente, mentalmente y socialmente (citado en Aguilar, 2008).

Entre las décadas de los años cuarenta y cincuenta en México las autoridades de salud creían todavía que la alimentación del mexicano era fuente de pobreza y atraso. Un consumo bajo en proteínas y calorías hacía que los trabajadores faltaran con frecuencia a trabajar o enfermaran constantemente. Por esta razón consideraron que la proteína animal en la dieta de los obreros haría que estos fueran mucho más productivos (Aguilar, 2008).

Estos prejuicios que, posteriormente, se convirtieron en políticas vigentes, en algunos casos hasta nuestros días fueron comunicados por la propaganda y la publicidad a los mexicanos de todos los niveles repetitivamente a lo largo de nuestra historia, convirtiéndose en parte del statu quo que prevalece en la mente de muchas personas todavía en la actualidad.

Como puede observarse, la alimentación y sus connotaciones constituyen un habitus que apresa a una parte de la población en un sitio social del que busca escapar continuamente 
a través de la adopción de elementos que además de ajenos le resultan dañinos, construyendo un campo en donde lo que está en juego es la pertenencia a uno u otro grupo social a través de la adopción de las costumbres, especialmente del estilo de vida que la publicidad genera sobre cierto tipo de alimentación (Soariano, 2008; Schwalb et al., 2014; Rivera et al., 2017; Rebeil, Flores, y Del Prado, 2018).

\section{METODOLOGÍA}

Se llevó a cabo una revisión de autores y sus diversos planteamientos que contrastan la historia paralela de la publicidad de los alimentos ultraprocesados y establecen una relación de los cambios de dieta con los fenómenos sociales, comerciales y políticos en España, Estados Unidos y México (De Deus et al., 2016; Popkin, 2012; Nestlé, 2002). Se llevó a cabo una aproximación documental del punto de vista de varios autores y desde la perspectiva de las Organizaciones Protectoras del Consumidor y Organizaciones Globales, con la finalidad de contrastar los escenarios planteados por estas respecto a las posibles causas de la epidemia de sobrepeso, obesidad y diabetes en diferentes países de bajo y medio ingresos, con especial énfasis en México con la finalidad de encontrar coincidencias y diferencias (Calvi1lo, 2018; Karageorgiadis, 2018; Macari, 2018).

\section{RESULTADOS}

Los resultados encontrados a la luz de los datos revisados son que si bien existe una relación importante entre los cambios sociales, políticos y comerciales entre los países, las campañas de comunicación desde la sociedad civil organizada señalan una cantidad mucho mayor de factores causales en la epidemia de sobrepeso, obesidad y diabetes que no son necesariamente los factores históricos exclusivamente, se mencionan: el ambiente obesogénico, las estrategias publicitarias de las multinacionales, los conflictos de intereses en cada país, los intereses económicos de los accionistas de las multinacionales por encima de la salud de las personas y la normalización de estilos de vida asociados a lo rápido, lo práctico, la dominación de clases, las connotaciones presuntamente positivas de ser moderno, práctico y tener tiempo para ser uno mismo. 


\section{CONCLUSIONES}

Aunque la publicidad de las grandes multinacionales posee un músculo político y económico sin paralelo en el mundo, las campañas de comunicación de las Organizaciones Protectoras del Consumidor parecen ir permeando poco a poco en la sociedad por medio de señalar los problemas que generan las multinacionales en su afán de construir mayor capital económico y político. Los fenómenos propios de cada país analizado han sido la base de la popularización de los productos ultraprocesados por encima de las dietas tradicionales, especialmente en el caso de México. A la luz de los datos, las campañas y estrategias de comunicación desde la sociedad civil organizada podrían ser un camino para la prevención y eventual solución de la epidemia de sobrepeso, obesidad y diabetes, pero el avance parece lento, ya que se requiere de un cambio desde la sociedad y no solamente desde las grandes empresas multinacionales o los tomadores de decisiones. Es una posibilidad empezar a tomar conciencia de que el problema de salud pública, que de acuerdo con las OPC causan las multinacionales y sus políticas, podría generar cambios en todos los diferentes stakebolders involucrados en el problema y la solución.

\section{REFERENCIAS}

Alianza por la Salud Alimentaria. (2018).El ambiente escolar obesogénico en México. México: Alianza por la Salud Alimentaria. https://doi.org/10.21149/spm.v58i4.8022

Alianza por la Salud Alimentaria. (2018). Publicidad dirigida a niños: Una infancia enganchada a la obesidad. México: Alianza por la Salud Alimentaria.

Aguilar, S. (2008). Alimentando a la nación: género y nutrición en México (1940-1960). Revista de Estudios Sociales, 28-40. https://doi.org/10.7440/res29.2008.02

Arribas, J. (1996). Nestlé: paradigma de la comunicación publicitaria, en Nestlé en España. 1905 2005. Reflexiones sobre su contribución al desarrollo económico y social. Barcelona: Nestlé.

Baraybar-Fernández, A.; Baños-González, M.; Barquero-Pérez, Ó.; Goya-Esteban, R., y De la Morena, A. (2017). Evaluación de las respuestas emocionales a la publicidad televisiva desde el neuromarketing. Comunicar, 19-28. https://doi.org/10.3916/c52-2017-02

Barragán, Guerra, y Villalpando. (2017). La economía de la experiencia y el marketing emocional: estrategias contemporáneas de comercialización. International Fournal of Good Conscience, 159170.

Brown, S. (2014). Animal house: Brand mascots, mascot brands and more besides. Fournal of Customer Behaviour, 77-92. https://doi.org/10.1362/147539214x14024779483519

Calvillo, A. (4 de julio de 2018). Publicidad engañosa: Retos para las ONG protectoras del consumidor (Del Prado, R., entrevistador). 
Canal Diabetes. (29 de agosto de 2018). Diabetes Infantil. Recuperado de https://www.canaldiabetes.com/mexico-mexico-desarrollara-diabetes-tipo-2/

De Deus, R.; Marçal, A.; Gea, A.; de la Fuente-Arriaga, C.; Martinez-Gonzalez, M. Á.; Sousa, A. C.; y Bes-Rastrollo, M. (12 de octubre de 2016). Ultraprocessed food consumption and risk of overweight and obesity: the University of Navarra Follow-Up (SUN) cobort study. Recuperado dehttps://academic.oup.com/ajcn/article/104/5/1433/4564389 https://oi.org/10.3945/ ajcn.116.135004

Días-Méndez, y González-Álvaez. (2013). La problematización de la alimentación: un recorrido sociológico por la publicidad alimentaria (1960-2010). Revista de Metodología de Ciencias Sociales, 121-146. https://doi.org/10.5944/empiria.25.2013.3800

El Poder del Consumidor. (2018). Explorando el ambiente escolar alimentario. Ciudad de México: El Poder del Consumidor.

Espinosa, A. M. (2017). La consolidación del ambiente obesogénico en México, 120-127.

Gracia, M. (1996). Paradojas de la Alimentación Contemporánea. Barcelona: Icaria.

Ibarra, L. (13 de julio de 2018). Publicidad engañosa: Retos para las ONG protectoras del consumidor (Del Prado, R., entrevistador).

Karageorgiadis, E. (17 de julio de 2018). Publicidad engañosa: Retos para las ONG protectoras del consumidor (Del Prado, R., entrevistador).

Macari, M. (19 de julio de 2018). Publicidad engañosa: Retos para las ONG protectoras del consumidor (Del Prado, R., entrevistador).

Nestle, M. (2002). Food Politics: How The Food Industry Influences Nutriotion and Helath. Los Angeles: University of California Press.

Olivares, A. M. (2018). Percepción de escolares chilenos de distinto nivel socioeconómico sobre la regulación de la publicidad de alimentos. Archivos Latinoamericanos de Nutrición, 88-96. https://doi.org/10.20960/nh.499

Organización Panamericana de la Salud. (2015). Alimentos y bebidas ultraprocesados en América Latina: tendencias, efecto sobre la obesidad e implicaciones para las politicas públicas. Washington, D.C.: Organización Panamericana de la Salud. https://doi.org/10.21149/spm.v58i5.8182

Pilgeram, R., y R. M. (2014). Good Food, Good Intentions: Where Pro-sustainability Arguments Get Stale in US Food Documentaries. Estados Unidos de América. https://doi.org/10.1080/ 17524032.2014.967706

Popkin, B. A. (2012). Global nutrition transition and the pandemic of obesity in developing countries. Nutrition reviews, 3-21.

Rebeil, Flores, y Del Prado. (2018). Videos engañosos de cereales ultraprocesados industrialmente: Métodos visuales para la conciencia social. Revista Latina. Tenerife.

Rivera, E.; Enciso, R.; Estrada, A. L.; y Fonseca, M. E. (2017). Publicidad televisiva y su influencia en el consumo de comida chatarra. Tepic, Nayarit, México.

Robinson, B. M. (2007). Effects of Fast Food Branding on Young Children's Taste Preferences. Fournal of Archives of Pediatrics y adolescent medicine, 792-797. https://doi.org/10.1001/archpedi.161.8.792 
Schwalb, Sanborn, Galarza, Garcia, y Kogan (2014). Comida chatarra, Estado y mercado. Lima, Perú.

Soariano, S. (2008). Trastornos de la conducta alimentaria: ¿Síndromes culturales? Bioéticay Debat, 9-12.

Sorscher, S. (17 de julio de 2018). Publicidad engañosa: Retos para las ONG protectoras del consumidor (Del Prado, R., entrevistador).

Taylor, A. L., y Jacobson, M. F. (2016). Carbonating the World: The Marketing and Health Impact of Sugar Drinks in Low- and Middle-income Countries. Washington, D.C.: Center for Science in the Public Interest.

The Public Health Advocacy Institute. (25 de septiembre de 2017). State Law Approaches to Address Digital Food Marketing to Youth. Recuperado de http://www.digitalads.org/sites/default/files/ publications/phai_digital_food_mktg_full_report.pdf

Turk, M. A., y E. F. (2015). What Is False, Deceptive or Misleading Advertising? Memphis, Tennessee, Estados Unidos de América.

World Health Organization (WHO). (2014). Global status report on noncommunicable diseases. Ginebra: WHO. 